\title{
Fault Detection Method for Energy Routing Nodes of Smart Grids Oriented to Electricity Information Security
}

\author{
Wenpeng $\mathrm{Li}^{\mathrm{a}}{ }^{*}$, Sance $\mathrm{Gao}^{\mathrm{a}}$, Renjie Ding ${ }^{\mathrm{a}}$, Yanjun $\mathrm{Hao}^{\mathrm{b}}$, and Cheng Yang ${ }^{\mathrm{b}}$ \\ ${ }^{a}$ Maintenance Branch of State Grid Inner Mongolia East Electric Power Co., Ltd., Tongliao, 028000, China \\ ${ }^{b}$ Beijing Zhongdian Nanrui Technology Co., Ltd., Beijing, 100000, China
}

\begin{abstract}
Current fault detection methods based on the immune mechanism for energy routing nodes of power grids have low peak strengths of estimated fault signals, resulting in a low probability of fault detection and fault location accuracy and a lack of fault isolation performance. A fault detection method for energy routing nodes of smart grids oriented to power information security is proposed. According to the fault characteristics of energy routing nodes of power grids, fault diagnosis criteria are given. The necessity and sufficiency of the fault diagnosis criteria are proven. The peak strengths of fault signals are estimated, and fault detection is realized. The fault line is judged by the natural frequency value of the fault traveling wave, and the traveling wave propagation that reflects the fault points is utilized. The intrinsic frequency value of the path can accurately calculate the fault distance and obtain the exact location of the fault. The fault isolation is accomplished by using the distributed power supply and combining it with the current power grid structure with the switch position. Experiments show that this method is superior to the current method in peak estimation strength, fault detection rate, and fault location, and the highest fault detection rate can reach $99 \%$. The implementation of the proposed method can effectively improve the current situation of power grid fault detection and provide a more scientific basis for the development of this field.
\end{abstract}

Keywords: smart grid; energy routing node; fault detection; power information security

(Submitted on October 28, 2019; Revised on November 30, 2019; Accepted on December 15, 2019)

(C) 2019 Totem Publisher, Inc. All rights reserved.

\section{Introduction}

The power grid system is in a complex working environment. The control parameters are complex and uncertain, which results in frequent failure of routing energy nodes. Effective fault detection of routing nodes in power grid systems is needed to improve the life cycle and automatic control performance of power grids. Smart grids refer to the intellectualization of power grids based on integrated, high-speed two-way communication networks [1]. In view of the continuous popularity of smart grids, fault detection has become an urgent problem to be solved [2-4]. Experts and scholars have explored solutions to this problem. Tong et al. studied an online identification method for fault lines in order to realize the fast and effective fault detection of power grids under a limited PMU layout [5]. According to the PMU layout, the power grid was divided into several areas. The characteristics of positive sequence, negative sequence, and zero sequence currents flowing into the fault area were analyzed when the fault occurred, and the fault area search method based on boundary PMU was studied. Based on virtual nodes, a pure fault equivalence model and a fault component voltage equation were constructed. Using the electrical quantity of each boundary node in a region, the overdetermined equations of fault component voltage were constructed. The least square method was used to solve the fault location of fault lines. The detection efficiency was high, but the accuracy of fault location of power grid was low.

Current methods for power grid fault detection cannot meet the needs of the development of modern power grid technology. A new automatic fault detection method for energy routing nodes in smart grid is needed to solve the problems of current related methods.

\footnotetext{
* Corresponding author.

E-mail address: wkr78d@163.com
} 


\section{Fault Detection Method for Energy Routing Nodes in Smart Grids}

\subsection{Fault Detection of Energy Routing Nodes in Smart Grids}

It is assumed that the smart grid $N$ has $n$ independent nodes and $b$ branches. If the current source excitation is properly added at $(m<n)$ accessible points, the whole grid can be regarded as an equivalent pure resistance network without the influence of inductance and capacitance, as shown in Figure 1.

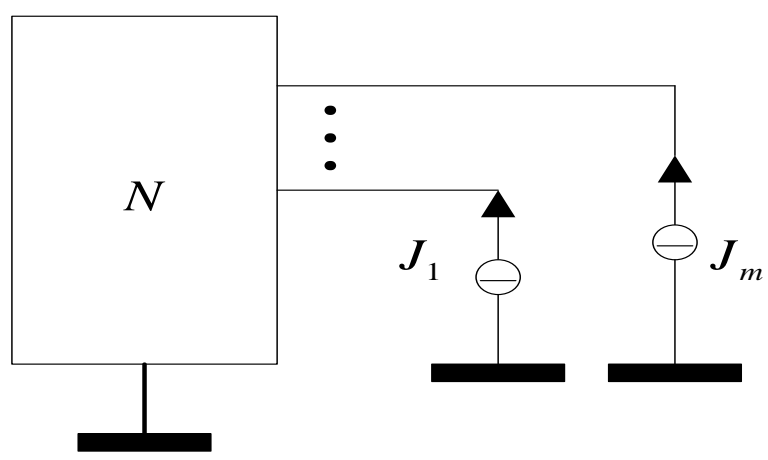

Figure 1. Equivalent diagram of grounding grid

According to Figure 1, the voltage equation of energy routing nodes in smart grids is obtained as follows:

$$
\left[Y_{n o}\right]\left[V_{n}\right]=\left[J_{m}\right]
$$

Where $Y_{n o}$ represents the admittance matrix of energy routing nodes in the network; $V_{n}$ represents the voltage vector of energy routing nodes in the grounding grid e; $V_{n}=\left[V_{1}, V_{m}\right]$, where $V_{1}$ and $V_{m}$ respectively represent interior contact points of the grounding grid and accessible point electric vector; and $J_{m}$ represents the current source excitation vector applied at $m$ accessible points, which is a known quantity.

The energy routing node in the grounding grid can be selected as the accessible testing point if it satisfies the following conditions: (1) the endpoint can be used for testing; (2) the sensitivity of the node voltage of the grounding grid to the change of parameters is not zero, and when any two independent faults occur simultaneously, the voltage on this node will not offset each other, and the estimation effect on the peak value of the fault signal of energy routing node can also be enhanced [6-7]. VM represents the calculated value of the voltage at the test point when the grounding grid is free of fault. In order to judge whether the smart grid $N$ fails or not, the measured values of accessible test points are usually used to compare with the calculated values when there is no fault.

The criteria for identifying faults of energy routing nodes in smart grids are as follows: the necessary and almost sufficient condition for no faults in smart grids is that the measured values of voltage at the accessible test points in the grids are equal to the calculated values without faults, i.e.,

$$
V_{m i}=V_{m i}^{(0)}
$$

Where $V_{m i}^{(0)}$ represents the voltage of the measured point, which cannot be buried underground in the power grid. When the network is not faulty, a current source excitation is applied to a certain accessible point. When $V_{m i}$ represents the fault of the power grid, the same current source excitation is applied to the same accessible point to measure the voltage value.

In summary, it can be concluded that when the network is fault-free, the voltage calculation value of the test point will be obtained as a function of $(i=1,2, \cdots)$. 


$$
V_{m i}^{(0)}=\phi_{0}+\left(\Delta \phi_{0}, J_{n}\right)
$$

Where $\phi_{0}$ represents the nominal value of grid parameters, $\Delta \phi_{0}$ represents the variation of external factors on grid resistance, and $J_{n}$ represents the external current source excitation, which is known. The reciprocal solution of the $V_{m i}^{(0)}$ value is the peak value estimation function of the fault signal.

The relationship between the accessible point voltage, the network parameters, and the external current source excitation can be expressed by Equation (3). If the ground network is fault-free, the value of $V_{m i}^{(0)}$ can be calculated by Equation (2). The function expression of the voltage measurement value $V_{m i}$ at the test point is to add the same current source excitation at the test point as in the case of no fault.

$$
V_{m i}=\left(\phi, J_{n}\right)
$$

Where $\phi$ represents the relationship function of the current change value of power grid parameters and is an undetermined value. If the change in $\phi$ does not exceed the range of change without cause, i.e., $\phi \in \phi_{0}+\Delta \phi_{0}$, then the power grid is still fault-free. The voltage measured at the test point will not exceed the fault-free calculation value, i.e., $V_{m i}=V_{m i}^{(0)}$. The necessity has been proven.

According to the basic conditions of the test points, the sensitivity of the node voltage in the grounding grid to the parameter changes is not zero. When the network internal parameters change, the voltage changes at the test points will not offset each other [8-9]. Assuming that the voltage value of the test point is $V_{m i}=V_{m i}^{(0)}$, it can be seen from Equations (3) and (4) that the variation of the parameters of the power grid cannot exceed the allowable range, that is, $\phi \in \phi_{0}+\Delta \phi_{0}$. Then, the power grid is fault-free, and the adequacy has been proven. The necessity and sufficiency proof are used to improve the accuracy of fault detection of energy routing nodes in power grids.

\subsection{Fault Location of Energy Routing Nodes in Smart Grids}

According to the test results obtained in Section 2.1, fault location is carried out by using the natural frequency of traveling waves.

The general circuit of the fault is judged. When a fault occurs in a power grid with known topology and fixed line length, the traveling waves generated by different line faults will propagate along different paths in the power grid, and the natural frequencies of the traveling waves formed by different transmission paths are different [10-11]. The natural frequencies of fault traveling waves propagating on a certain path $D$ are as follows:

$$
f=\frac{(h \times v)}{(4 \pi \times D)}
$$

Where $D$ represents the propagation path of the fault traveling wave, $h$ represents the boundary condition of the fault traveling wave propagation, and $v$ represents the propagation speed of the traveling wave, which is determined by the refraction and reflection angle at the discontinuous point of wave impedance on the line.

When the propagation path $D$ of the fault traveling wave is composed of only sound lines, the propagation boundary condition $h$ and the propagation velocity $v$ hardly change with the frequency because the distance of the propagation path is a definite value, which can be approximately regarded as a constant. According to Equation (5), the natural frequency of the fault traveling wave on the propagation path $D$ can be considered as a fixed value [12]. When the fault traveling wave on the propagation path $D$ contains the fault point, because the distance of the propagation path is related to the fault distance $d$, the natural frequency value of the traveling wave reflecting the fault propagation path is related to the fault distance $d$ and is no longer a fixed value. 
When a fault occurs on the installation line of the fault location device ( $L_{1}$ in Figure 2 ), the natural frequency component of the traveling wave reflecting the fault distance $d$ in the fault traveling wave signal is much larger than the natural frequency component reflecting other propagation paths, and because of the fault distance $d<L_{1}$, its frequency value will be greater than the natural frequency value reflecting $L_{1}$. In this way, the natural frequency of the traveling wave reflecting the fault distance can be easily identified, as shown in Figure 3.

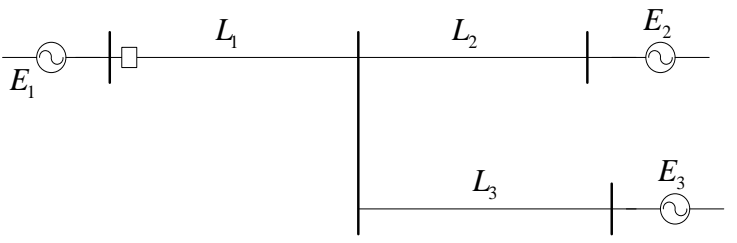

Figure 2. Schematic diagram of transmission path of energy node in power grid

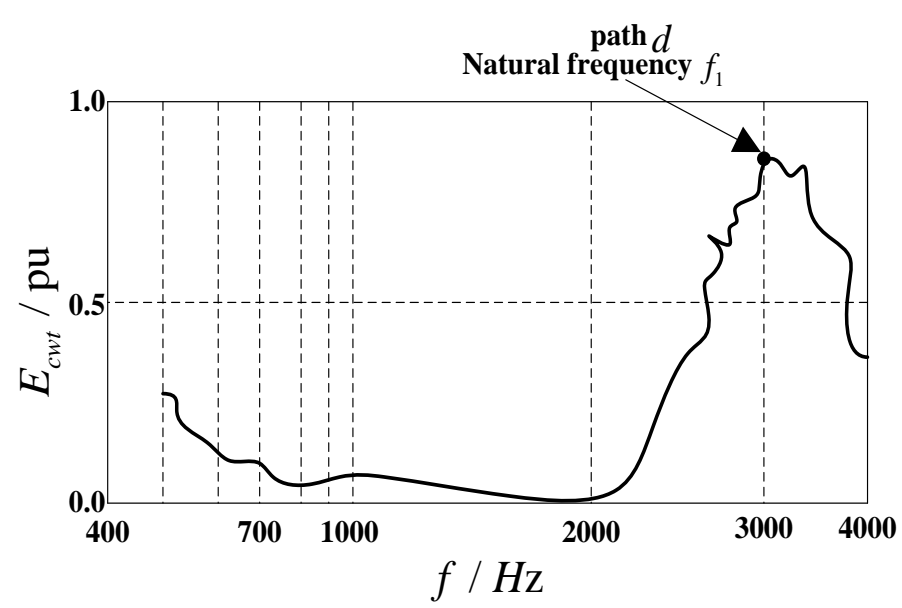

Figure 3. Spectrum of fault traveling wave at $L_{1}$

On the basis of judging the fault line, the fault distance can be calculated according to the principal component value of the traveling wave's natural frequency, which reflects the propagation path of the fault point [13]. When a fault occurs on $L_{1}$, the estimation formula of the fault distance $d$ is as follows:

$$
d=\frac{\left[\left(\theta_{x}+2 k \pi\right) v_{d}\right]}{\left(4 \pi f_{d}\right)}
$$

Where $\theta_{x}$ represents the phase angle of the reflection coefficient, $2 k \pi$ represents the phase change of the fault traveling wave, $v_{d}$ represents the propagation speed of the traveling wave in the $d$ section of fault distance, and $f_{d}$ represents the natural frequency value.

When a fault occurs in $L_{2}$ or $L_{3}$, the formula for calculating the fault distance is expressed as

$$
L_{1}+d=\frac{\left(\theta_{x}+2 \varphi+2 k \pi\right) v_{d}}{4 \pi f_{d}}
$$

Where ${ }^{\varphi}$ represents the phase angle of the refractive index. The catadioptric angle and traveling wave velocity in Equations (6) and (7) are both values under the natural frequency $f_{d}$.

Aiming at the realization of fault location, a fault location method based on the traveling wave propagation path and its natural frequency is proposed [14], which includes the determination of the fault line and the accurate calculation of fault 
distance.

\subsection{Fault Isolation of Energy Routing Nodes in Smart Grids}

In order to better realize the repair of power grid faults, based on the fault location in Section 2.2 and combined with the current network structure with the switch position status, fault isolation can be achieved.

Due to the variability of distribution network operation mode and the existence of DG (distributed generation), the switches that need to be jumped out in the same fault section may not be the same each time. Therefore, on the basis of judging the fault section, it is necessary to combine the current network topology structure to determine the switch that needs to be jumped off to isolate the fault. The fault isolation method is implemented by matrix operation [15-16], which requires defining a switch, a line correlation matrix $Q$, and a tripping switch vector $a$. Among them, $Q$ is used to describe the corresponding relationship between the switch and the line. $a$ is calculated by $P$ (the fault section matrix obtained in Section 2.2) and $Q$, which is used to describe the switch that needs to be jumped out to isolate the fault.

$Q$ is a square matrix of order $M$. The element $q_{i j}$ in $Q$ is defined as follows:

$$
q_{i j}= \begin{cases}1, & \text { Branch } i \text { is directly connected to node } j \\ 0, & \text { Branch } i \text { is not directly connected to node } j\end{cases}
$$

The tripping switch vector $D$ is obtained by multiplying $P$ and $Q$.

$$
D=P \times Q
$$

Where, when the element in $D$ is 1 , the switch corresponding to the consistent element is the switch that needs to be jumped.

\section{Experimental Results and Analysis}

In order to verify the performance of the proposed method in fault detection of routing nodes in automatic control power grids, simulation experiments are needed. Computer A and computer B are used for routing communication in power grids. The computer configuration information in the simulation environment is as follows: the model of computer A is Dell 2210 b, with processor Intel Core 2 Duo $1.80 \mathrm{GHz}, 1 \mathrm{G}$ memory, and main frequency DDR 2 667; the model of computer B is Lenovo KM400, with processor AMD Athlon $1.83 \mathrm{GHz}, 2 \mathrm{G}$ memory, and main frequency DDR 333. The protocol of power grid communication adopts a high-efficiency, reliable, and low-cost MAC protocol. The model of power data transmission of automatic control power grids is constructed by using the model of IEEE 802.15.4 routing protocol. The experiment is completed by the Visual $\mathrm{C}$ simulation tool. The routing samples of the routing nodes of the automatic control power grid are averagely sampled, and there are 1,000 routing node samples. The distribution node model for initial routing of the power grid is obtained as shown in Figure 4.

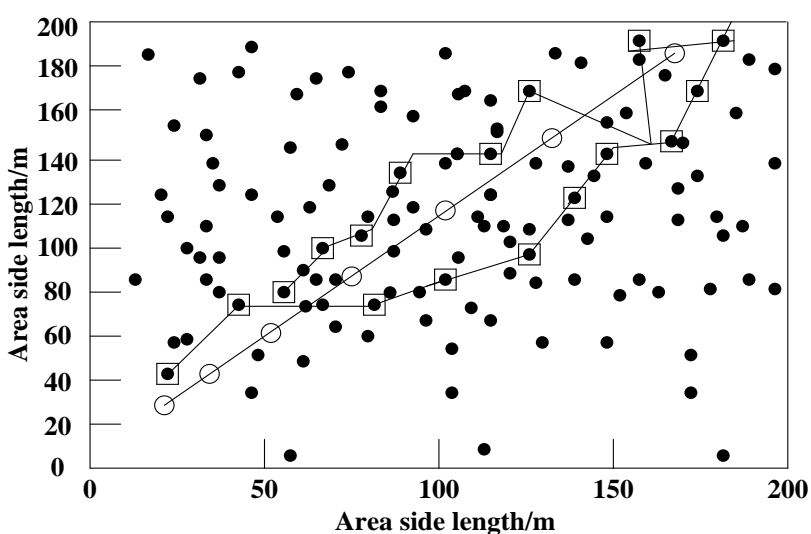

Figure 4. Distributed node model for initial routing in power grids 
According to the above simulation environment and routing distribution structure, node fault detection is carried out, and fault signal samples are collected. The samples are divided into fault state and normal receiving and receiving state. Each state sample is 100 , totaling 400 samples. The samples are divided into a training set and a testing set according to a 4:1 ratio. The beam model of the direct signal from the fault source of the routing node is obtained as shown in Figure 5.

According to the model given in Figures 4 and 5, the fault detection effect of the proposed method is verified. From Figure 5, it can be seen that the power spectrum intensity of the direct signal from the fault source of the routing node fluctuates greatly. The fault detection method based on the immune mechanism for the fault routing node of the power grid is used to verify the effectiveness of each method in estimating the peak value of the fault signal intensity of the energy routing node. The experimental results are shown in Figure 6.

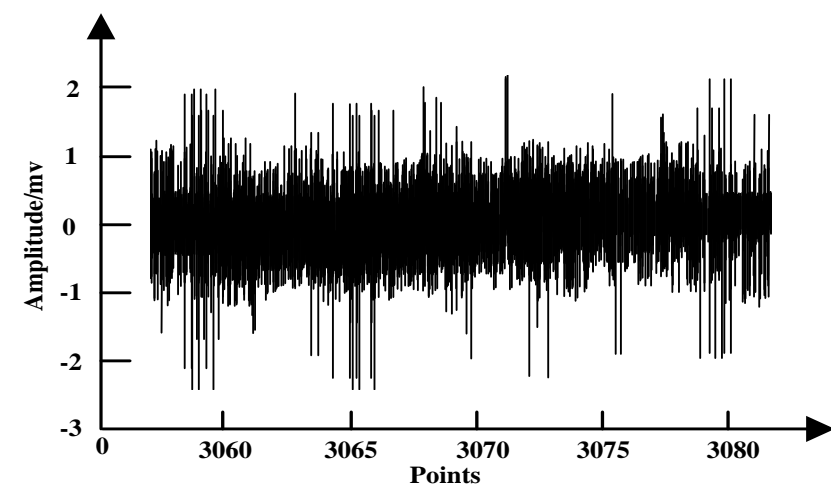

Figure 5. Beam model of direct signal from the fault source of routing nodes

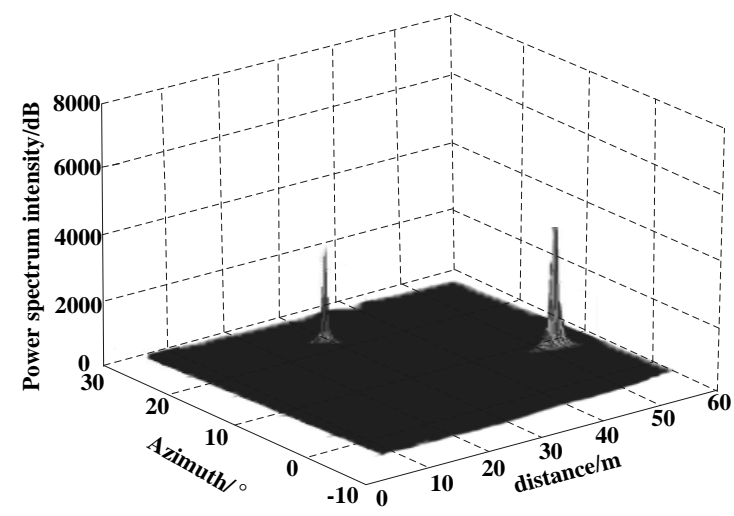

(a) Estimation results of fault signal intensity of energy routing nodes in power grids based on immune mechanism

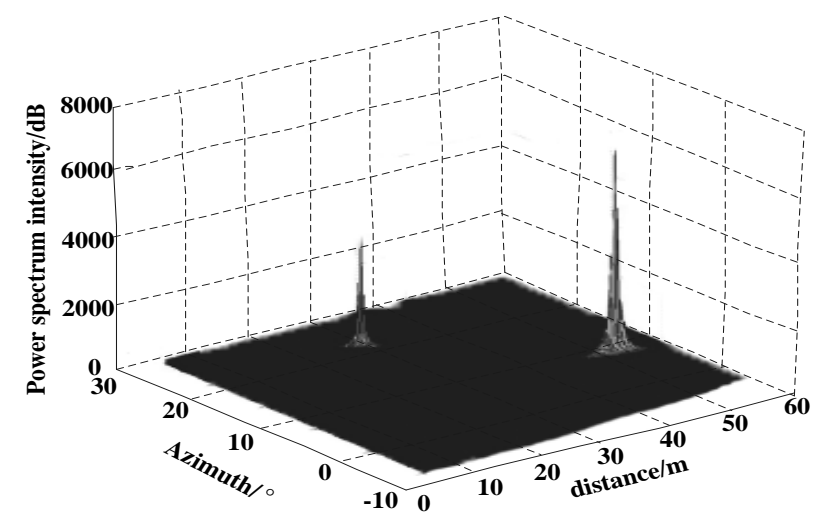

(b) Estimation results of fault signal intensity of energy routing nodes in power grids based on the proposed method

The analysis of the experimental results of Figure 6 shows that the proposed method has a strong peak value estimation effect. The estimated peak value is 6,000, which can effectively detect the faults of energy routing nodes in smart grids. The experimental results are shown in Figure 7 with the accurate probability of fault detection as the test index. As can be seen from Figure 7, this paper improves the fault detection accuracy of energy routing nodes in smart grids by proving the necessity and sufficiency. The performance is also superior. The fault detection accuracy can reach $99 \%$, while the fault detection accuracy of energy routing nodes based on the immune mechanism can reach $84 \%$. 


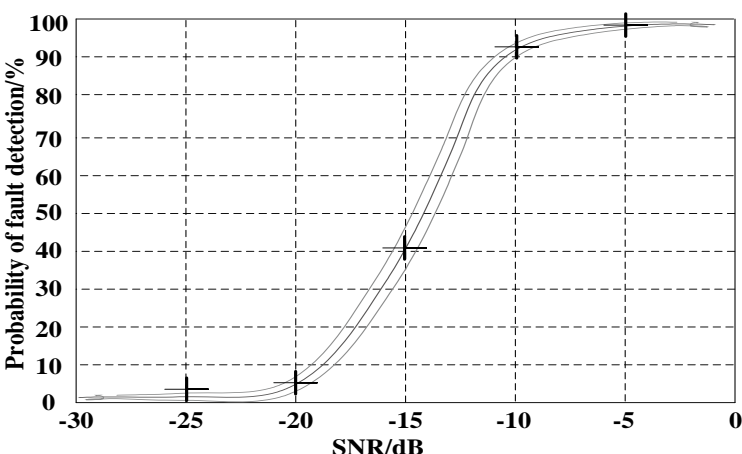

(a) Accuracy of fault detection for energy routing nodes in power grids based on the proposed method

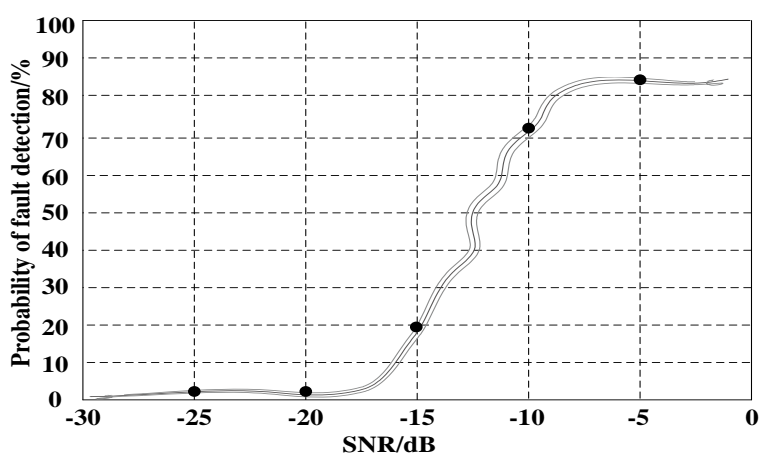

(b) Accuracy of fault detection for energy routing nodes in power grids based on immune mechanism

Figure 7. Comparison of the accuracy of power grid fault detection by different methods

Figure 8 shows the comparison results of fault location accuracy of energy routing nodes in power grids by different methods. Figure 8(a) is the experimental model.

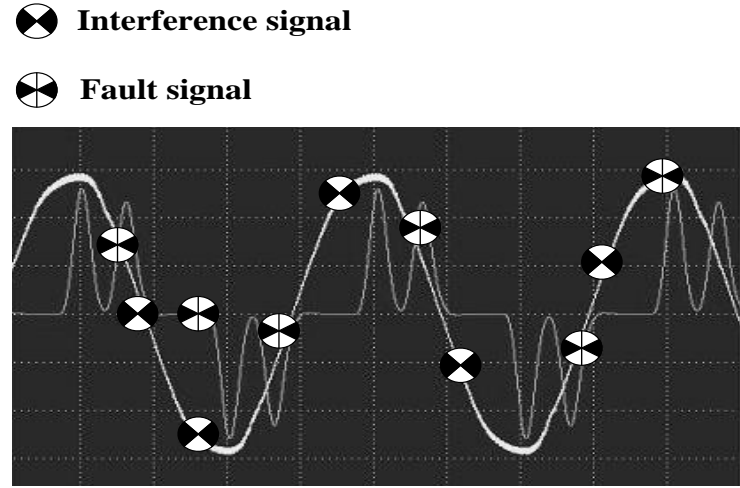

(a) Experimental model

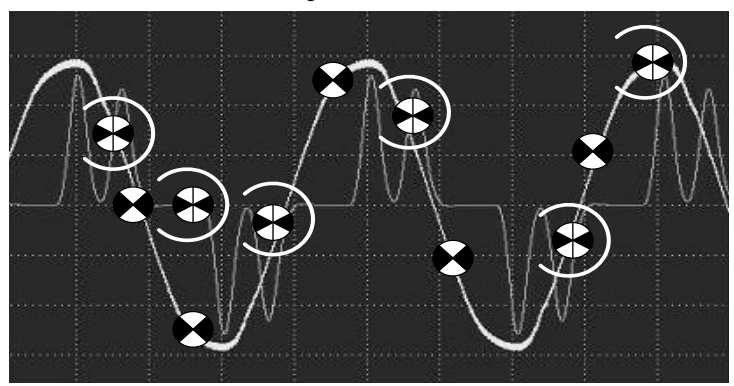

(c) Recommended method

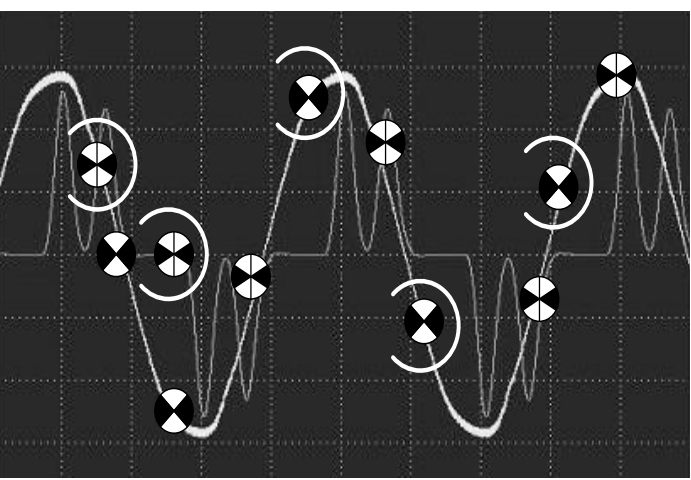

(b) Immune mechanism based approach

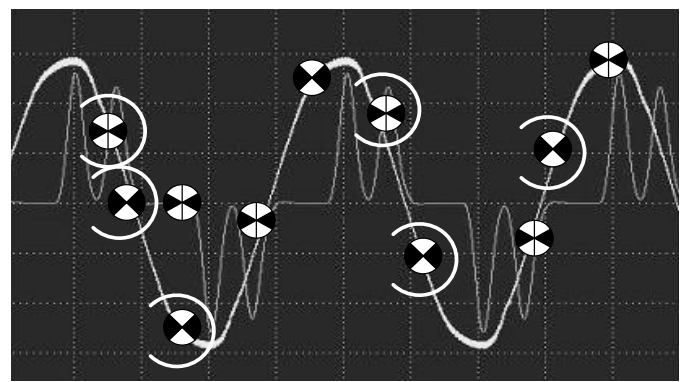

(d) Finite PMU method

Figure 8. Accuracy comparison of different methods for fault location of power grids

As it can be seen from Figure 8, the proposed method can accurately locate the fault detection results by utilizing the characteristic that the fault traveling waves generated by different line faults will propagate along different paths in the power grid, and the natural frequencies of the traveling waves formed by different transmission paths are different. The fault location accuracy of energy routing nodes based on the immune mechanism and that based on limited PMU are low. In particular, the fault location accuracy of energy routing nodes based on limited PMU is far below the technical requirements of fault detection.

\section{Conclusions}

Under the current hardware implementation conditions of power grids, the energy routing nodes of power grids have frequent faults, which requires effective fault detection [17-18]. This paper achieves the intended purpose through efficient fault detection, accurate location, and isolation. Experiments show that the proposed method not only improves the existing problems of low detection rate and poor fault location accuracy, but also isolates the faults and has high reliability. The 
formulation of the future research direction is as follows:

(1) In order to further improve the quality of fault detection, interference removal can be carried out in advance.

(2) To improve the process of fault detection, the contents of fault repair should be added.

\section{Acknowledgments}

This work is supported by the Second Batch of Service Bidding and Purchasing of Mengdong Electric Power in 2018: Development and Application of Online Monitoring and Fusion Platform for Ultra-high Voltage Transmission Line based on Big Data (No. MDZB-07182C).

\section{References}

1. Y. Lin, X. Zhu, Z. Zheng, Z. Dou, and R. L. Zhu, "The Individual Identification Method of Wireless Device based on Dimensionality Reduction and Machine Learning,” Journal of Supercomputing, Vol. 75, No. 6, pp. 3010-3027, 2019

2. Y. Lin, C. Wang, J. X. Wang, and Z. Dou, "A Novel Dynamic Spectrum Access Framework based on Reinforcement Learning for Cognitive Radio Sensor Networks," Sensors, Vol. 16, No. 10, pp. 1675, 2016

3. J. Sneath and A. D. Rajapakse, "Fault Detection and Interruption in an Earthed HVDC Grid using ROCOV and Hybrid DC Breakers," IEEE Transactions on Power Delivery, Vol. 31, No. 3, pp. 973-981, 2015

4. P. M. Papadopoulos, L. Hadjidemetriou, E. Kyriakides, and M. M. Polycarpou, "Robust Fault Detection, Isolation and Accommodation of Current Sensors in Grid Side Converters," IEEE Transactions on Industry Applications, Vol. 10, No. 99, pp. $1-10,2016$

5. X. Y. Tong, R. H. Wang, H. B. Wang, and W. Lian, "Online Detection Algorithm of Power System Fault using Limited PMUs," Power System Protection and Control, Vol. 44, No. 19, pp. 49-55, 2016

6. A. Brancaccio and R. Solimene, "Fault Detection in Dielectric Grid Scatterers," Optics Express, Vol. 23, No. 7, pp. 8200-8215, 2016

7. T. Neumann, T. Wijnhoven, G. Deconinck, and I. Erlich, "Enhanced Dynamic Voltage Control of Type 4 Wind Turbines During Unbalanced Grid Faults," IEEE Transactions on Energy Conversion, Vol. 30, No. 4, pp. 1650-1659, 2015

8. G. Yang and S. Liu, "Distributed Cooperative Algorithm for k-M Set with Negative Integer k by Fractal Symmetrical Property," International Journal of Distributed Sensor Networks, Vol. 4, pp. 1-7, 2014

9. U. M. Choi, J. S. Lee, F. Blaabjerg, and K. B. Lee, "Open-Circuit Fault Diagnosis and Fault-Tolerant Control for a Grid-Connected NPC Inverter," IEEE Transactions on Power Electronics, Vol. 31, No. 10, pp. 7234-7247, 2015

10. F. B. Youssef and L. Sbita, "Sensors Fault Diagnosis and Fault Tolerant Control for Grid Connected PV System," International Journal of Hydrogen Energy, Vol. 42, No. 13, pp. 8962-8971, 2016

11. C. Guo, Y. Liu, C. Zhao, X. Wei, and W. Xu, "Power Component Fault Detection Method and Improved Current Order Limiter Control for Commutation Failure Mitigation in HVDC," IEEE Transactions on Power Delivery, Vol. 30, No. 3, pp. 1585-1593, 2015

12. N. L. Georgijevic, M. V. Jankovic, S. Srdic, and Z. Radakovic, "The Detection of Series Arc Fault in Photovoltaic Systems based on the Arc Current Entropy," IEEE Transactions on Power Electronics, Vol. 31, No. 8, pp. 5917-5930, 2015

13. L. Zhu, J. Li, Y. Liu, and S. Ji, "Initial Features of the Unintended Atmospheric Pressure DC Arcs and Their Application on the Fault Detection," IEEE Transactions on Plasma Science, Vol. 45, No. 4, pp. 742-748, 2017

14. M. Dong, H. Dong, L. Wang, J. Yang, L. Li, and Y. Wang, "A Simple Open-Circuit Detection Strategy for a Single-Phase Grid-Connected pv Inverter Fed from Power Optimizers," IEEE Transactions on Power Electronics, Vol. 6, No. 99, pp. 1-1, 2017

15. Y. Fan, C. Li, W. Zhu, X. Zhang, L. Zhang, and M. Cheng, "Stator Winding Interturn Short-Circuit Faults Severity Detection Controlled by OW-SVPWM Without CMV of a Five-Phase FTFSCW-IPM," IEEE Transactions on Industry Applications, Vol. 53, No. 1, pp. 194-202, 2016

16. M. M. A. Mahfouz and M. A. H. El-Sayed, "Smart Grid Fault Detection and Classification with Multi-Distributed Generation based on Current Signals Approach," IET Generation Transmission amd Distribution, Vol. 10, No. 16, pp. 4040-4047, 2016

17. M. Liu, S. Liu, W. Fu, and J. T. Zhou, "Distributional Escape Time Algorithm based on Generalized Fractal Sets in Cloud Environment," Chinese Journal of Electronics, Vol. 24, No. 1, pp. 124-127, 2015

18. S. Liu, "Introduction of Key Problems in Long-Distance Learning and Training," Mobile Networks and Applications, Vol. 24, No. 1, pp. 1-4, 2019 